\title{
Type and token bigram frequencies for two-through nine-letter words and the prediction of anagram difficulty
}

\author{
David Knight • Steven J. Muncer
}

Published online: 17 March 2011

(C) Psychonomic Society, Inc. 2011

\begin{abstract}
Recent research on anagram solution has produced two original findings. First, it has shown that a new bigram frequency measure called top rank, which is based on a comparison of summed bigram frequencies, is an important predictor of anagram difficulty. Second, it has suggested that the measures from a type count are better than token measures at predicting anagram difficulty. Testing these hypotheses has been difficult because the computation of the bigram statistics is difficult. We present a program that calculates bigram measures for two-to nine-letter words. We then show how the program can be used to compare the contribution of top rank and other bigram frequency measures derived from both a token and a type count. Contrary to previous research, we report that type measures are not better at predicting anagram solution times and that top rank is not the best predictor of anagram difficulty. Lastly we use this program to show that type bigram frequencies are not as good as token bigram frequencies at predicting word identification reaction time.
\end{abstract}

Keywords Anagram·Token · Type - GTZero

\section{Knight}

Applied Psychology, Department of Psychology

University of Durham,

Thornaby-on-Tees,

Durham TS17 6BH, UK

\section{S. J. Muncer $(\square)$}

Clinical Psychology Programme, Teesside University,

Middlesbrough, TS1 3BA, UK

e-mail: S.Muncer@tees.ac.uk
Type and token bigram frequencies for two- through nine-letter words and the prediction of anagram difficulty

In this article, we describe a computer program that calculates bigram frequencies (two-letter sequences) for two- through nineletter words and letter strings derived from a token count and a type count. This program is unique in calculating measures for both type frequencies and token frequencies from the same word corpus and, thus, allows for a comparison of the importance of the different frequencies, which are defined below. The program calculates all of the major bigram frequency measures that have been proposed as being important in predicting anagram solution and word identification tasks. All of these bigram frequency measures are based on positional frequency counts of a bigram in a particular position in a word. The summed bigram frequency (SBF), for example, is the aggregate frequency of each bigram in each position. Examples of the calculation of all of the important bigram measures will be given below.

In order to demonstrate the use of the program, we reexamined research on five-letter anagrams by Novick and Sherman $(2004,2008)$ that challenged previous explanations of anagram solution in two major ways. First, they suggested that bigram frequency measures calculated as a type measure of frequency are more important than those calculated as a token measure of frequency. Most previous anagram research has used token bigram frequencies provided by Mayzner and Tresselt (1965). They provided bigram counts derived from 100 samples of 200 words taken from a variety of newspapers, magazines, and both fiction and nonfiction books. They counted each instance of 
every bigram in three- to seven-letter words in this corpus in each possible position. So, for example, every time the word because appeared, the initial bigram be received a count of 1 in the first position, and so on. If there were 20 appearances (tokens) of because in the word samples, be in the first position received a frequency of 20 from this word alone. A type measure of bigram frequency indicates the number of different words that contain the targeted bigram, rather than the number of tokens with the targeted bigram. So the be in the word because has a type count of 1 . Solso, Topper, and Macey (1973) made a similar distinction between what they called bigram frequency and bigram versatility. They used the example of the bigram of to demonstrate that there will be differences between bigram frequency (token) and versatility (type) measures. The bigram of has a relatively high token frequency in the English language, but this is largely based on the frequency of the word of.

Novick and Sherman (2004) suggested that a type frequency would be a better predictor of anagram difficulty because it was not confounded with word frequency. Novick and Sherman (2004) also pointed out that the Mayzner and Tresselt (1965) tables were derived from only a small subset of the words in the English language. For example, there were only 856 different five-letter words. Accordingly they produced a set of type frequencies for five-letter English words based on 2,550 different words, which were used in their program.

Novick and Sherman (2004) also proposed that a new bigram frequency measure called top rank, which was calculated from the type frequencies used in their computer program, would be a better predictor of anagram solution. They compared top rank against the SBF measure derived from Mayzner and Tresselt's (1965) token frequencies and found that the top rank measure was a better predictor of anagram solution time. Novick and Sherman (2004) concluded "that type-based bigram frequency is a better predictor of the difficulty of anagram solution than is tokenbased frequency" (p.397).

The relative importance of type and token measures is the subject of a controversial debate in word processing (Hofmann, Stenneken, Conrad, \& Jacobs, 2007). There is, however, no doubt that its resolution will be very important for current and future models of word recognition in general (Conrad, Carreiras, \& Jacobs, 2008). We believe that Novick and Sherman's (2004) conclusion that type frequencies were more important than token measures is misleading,since their study confounded two variables. They showed that a type frequency (top rank) was more important than a token frequency (SBF) but did not make the appropriate comparison between a type and token frequency measure of both SBF and top rank. They also ignored several other bigram frequency variables that have been considered more important in anagram solution than SBF and that may also be more important than their top rank measure.

Previous anagram research has suggested a multitude of variables that influence the difficulty of anagrams. Some of these are related to features of the solution word, such as word imagery, concreteness of the word, familiarity, objective frequency, age of acquisition, meaningfulness, number of vowels, starting letter, and some bigram frequency measures. Others are related to the composition of the anagram, such as the similarity of the word and anagram, and the pronounceability of the anagram (Gilhooly \& Johnson, 1978). Later research has suggested that the bigram frequency measures are the most important features of the solution word in predicting anagram difficulty (Gilhooly \& Johnson, 1978; Mendelsohn, 1976; Mendelsohn \& O'Brien, 1974).

The three main frequency measures that have been proposed for use in the prediction of anagram difficulty are the SBF(Mayzner \& Tresselt, 1959), bigram rank (BR; Mendelsohn \& O'Brien, 1974), and greaterthanzero (GTZero; Mendelsohn, 1976). Novick and Sherman (2004) included only SBF and their new top rank measure in their analysis. The starting frequencies for all of these measures have usually been provided by the Mayzner and Tresselt (1965) tables, which give frequencies for each bigram, in each position, for words between three and seven letters long. As was mentioned earlier, SBF is the aggregated frequency of each bigram in each position. For example, for the word light, we would calculate the frequency of $l i$ in the first two positions of a five-letter word $(l i=36)$, the frequency of ig in positions two and three $(i g=101)$, the frequency of $g h$ in positions three and four $(g h=94)$, and the frequency of $h t$ in positions four and five $(h t=95)$. These frequencies are then summed to produce a token SBF for light of 326.

Top rank is based on a comparison of the SBF, which is the sum of the frequencies of a word's successive bigrams. A word is top ranked if it has the highest SBF of the 120 possible combinations of bigrams for a five-letter word. Novick and Sherman's $(2004,2008)$ top rank was based on a comparison of the SBFs of the type frequencies of the words used in their study, but it is possible to produce top rank from token frequencies as well.

BR (Mendelsohn \& O’Brien, 1974) can also be calculated from the Mayzner and Tresselt (1965) tables, although it is not easily calculated by hand,since it requires the completion of a bigram frequency matrix for all of the bigrams (all combinations of two letter sequences) in a word in all of the possible positions. There are 80 cells in the matrix, since there are four bigram positions in a fiveletter word (see above), and there are 20 possible different combinations of bigrams. BRis the total number of cell 
entries in the bigram frequency matrix that have higher frequencies than the four correct cell frequencies. For example, the bigram frequency matrix for the word beach shows that in the correct position, be has a frequency of 41 , $e a$ in the correct position a frequency of $134, a c$ of 51 , and ch of 126. In the matrix for beach there are five incorrect bigrams that have higher values than $b e$, One incorrect bigram has a higher frequency than $e a$, five exceed $a c$, and one exceeds the value of $c h$. Thus, the BR for beach is 12 . The incorrect bigram with the highest frequency, which is higher than both ea and $c h$, is he in the second and third positions (263). Higher numbers for BR thus indicate more competition for the locations of the letters and, therefore, greater difficulty of solution.

GTZero (Mendelsohn, 1976) is also calculated from the bigram frequency matrix. GTZero is the total number of bigrams in a word with a frequency greater than zero in the bigram frequency matrix. For example, for the anagram igthl (light), hg, ht, hl, gt, tg, tl, lh, lg, and lt would all have a frequency of 0 in the first two positions. The measure is a development from Ronning's (1965) rule-out theory of anagram solution, which proposes that anagrams with a low number of bigram combinations that can be "ruled-out" of consideration will be harder to solve. The more nonzero entries there are, the greater the possible competing solutions, which makes the anagram harder to solve (Mendelsohn, 1976). Again, for any five-letter word, there are 80 possible cells in the bigram frequency matrix, since each bigram can appear in four positions. That is, in the word light, the bigram $l i$ can appear in competing words in the first and second positions, the second and third positions, the third and fourth positions, or the fourth and fifth positions, and there are 20 possible bigram combinations in a five-letter word. The GTZero for the word light is 33 .

Novick and Sherman (2004) produced a computer program that calculates SBF and their new bigram measure, top rank, for five-letter words. The program, however, does not calculate GTZero or BR. They did not include these bigram frequency measures in their analysis but included only SBF, which fewer researchers have argued predicts anagram difficulty. Furthermore, they did not include a top rank measure from a token frequency count. Our reanalysis will correct these omissions by examining the relative importance of all bigram frequency measures.

It is possible to calculate a type and token count for all of the bigram frequency measures apart from GTZero, because GTZero is calculated by counting the nonzero bigram frequencies in the bigram frequency matrix without considering their size, as do other measures. So if a cell has a frequency above 1, it will contribute to the GTZero score in the same way from either a bigram frequency matrix based on type counts or one based on token counts. For GTZero, the distinction between type and token frequen- cies is irrelevant conceptually. It is, however, not possible to produce both type and token frequencies even for the other variables from either the program described by Novick and Sherman (2004) or the tables of token frequency described in Mayzner and Tresselt (1965), since neither provides an alternative. Furthermore, since these two studies are based on different word corpora, any differences in predictive ability might be based on differences in the size and quality of the word corpora. We have, therefore, used the bigram frequencies provided by Solso and Juel (1980) to derive a set of type and token statistics from the Kučera and Francis (1967) corpus. Solso and Juel referred to the token frequencies as positional frequencies, which is defined as how many times a bigram appears in a specific position per one million words. They called type bigram frequencies versatilities, which is defined as how many different words a bigram appears in, in a specific position, per one million words.

Novick and Sherman (2004) noted that one of the limitations of the Solso and Juel (1980) norms was that only a printed version of them existed and that calculating these bigram measures from frequency tables is both a laborious and a potentially error-prone process. For example, Seidenberg $(1987,1989)$ argued that the evidence for the syllable as a functional unit during reading could be explained by the fact that bigram frequencies at the boundary of two syllables are lower than intrasyllabic bigram frequencies. Readers could, therefore, be sensitive to this bigram trough, in which bigram frequencies at a syllable boundary are lower than those for the preceding and following bigrams, rather than to syllables per se. Rapp (1992) conducted an experiment to test the bigram trough hypothesis that required the calculation of bigram frequencies at, before, and after a syllable boundary. In Rapp's experiment, the bigram frequencies were calculated incorrectly for 4 out of 117 words. Errors are even more likely in the calculation of more complicated statistics,such as BR and GTZero, that require the completion of a frequency matrix. For example, in Gilhooly and Johnson's (1978) study of the impact of 12 variables on anagram solution, their token bigram frequency measure, called GTZero,was incorrect in 38 of 80 cases.

Our computer program calculates all relevant bigram frequency statistics that have been used in anagram research in both type and token form. We used this program to calculate all relevant bigram measures for the data reported by Novick and Sherman (2004) and others. This enabled both a thorough comparison of the importance of the type and token distinction and an evaluation of the different bigram frequency measures in predicting anagram difficulty. This comparison was not confounded by size and quality of word corpora,since both counts were based on the same corpus. Novick and Sherman (2004) noted that the 
calculation of bigram and other sublexical frequencies has been important "across a variety of fields over at least the past 40 years" (p.397). The importance of sublexical frequency measures in general language processing is also recognized (Aichert \& Ziegler, 2005; Hofmann et al., 2007). Therefore, we also examined the relationship between the various bigram measures and time taken on a lexical decision task, in order to demonstrate the wider applications of the program.

\section{The program}

There are 577 different bigrams in the Solso and Juel (1980) tableswith a frequency count for both word tokens and word types in each bigram position for words between two and nine letters long. Each bigram can appear in numerous positions dependent on word length. So for a two-letter word, there is only one bigram position, first and second, but for a five-letter word, a bigram can appear in four positions, and so on. Our computer programcalled Bigram Calculator for Solso and Juel, computes all of the major bigram frequency statistics for any letter string with a length of from two to nine letters, using the Solso and Juel tables. These include the simplest statistics, such as the bigram frequency in each position, which could be used to calculate bigram troughs, and also SBF. It also calculates the more complicated statistics, such as BR, GTZero, and the top rank measure suggested by Novick and Sherman (2004). In order to calculate top rank, it has to compute the SBFs of the 120 possible orders of the five letters in the letter string and then rank them. Our program refers to this ranking as the likelihood $\operatorname{rank}(\mathrm{LR})$, since from a bigram frequency perspective, it is a measure of the likelihood of that combination of letters. The program also gives the lowest ranked combination of letters and the SBF for this lowest combination.

Our MS Windows-based program consists of six files (compressed as a single zip file) that can be downloaded from http://spider.dur.ac.uk/bical. The "Table.csv" file consists of position-sensitive token and type bigram norms taken from Solso and Juel (1980) for two- to nine-letter words. The "BiCal.exe" file is the main executable program file and provides the bigram frequency for each position for the correct solution, SBF, GTZero, BR, LR, and top rank based on these norms. The final four files make up the help system, which gives directions on running the program and can be accessed either from within the program from the "Help" menu or by launching the "BiCal.hlp" file.

In order to run the program, a word is entered into the Input box and the "Go" button is clicked. The token bigram frequency totals for each bigram, their sum (SBF), BR, GTZero, and LR appear on the left, and the type frequencies on the right. Words with an LR of 1 would be given a top rank score of 1 , and the rest 0 . For the word light, the token SBF is 8361 , the BR is 12 , and the LR is 8 , whereas the type SBF is $89, \mathrm{BR}$ is 51 , and LR is 8 . The GTZero from both the type and token frequencies is 49 and will always be identical, as was explained earlier. GTZero, however, is likely to be affected by the size of the corpus from which it is calculated; the more words that are included, the greater the likelihood that one of them will have a bigram in each position. For example, for the word light, the GTZero from Solso and Juel (1980) is 49; calculated from Novick and Sherman's (2004) type frequencies, it is 45 , whereas it is 33 when calculated from Mayzner and Tresselt's (1965) smaller token corpus. It is possible, of course, that if a corpus is very large, it will include many words that are unfamiliar to most people, and this may mean that it is less effective at predicting anagram solutions.

The program can take several seconds to calculate results for longer words. This is due to the number of calculations necessary to work out the LR, since the number of possible permutations of the letters in the word increases exponentially with the word length. For instance, a nine-letter word consists of 362,880 different possible letter combinations, and these contain a total of 2,903,040 bigrams. Each of these has to be looked up in the Solso and Juel (1980) statistics table in order to calculate the SBF for that permutation of letters, and then the results are ranked.

The program can also perform batch processing on a list of words. The list of words to be processed should be saved as a standard text file with each word on a separate line. This list of words can then be loaded into the program by going to the "File" menu and selecting the "Load Word List" option. The program will scan the list and will automatically reject any words that are not from two to nine characters in length (and will notify the user of this), although it will continue processing any subsequent words. After this, the program prompts for the name of an output results file (again, a standard text file). The program calculates the various results/scores for each of the valid words in the list and then saves these into the results file. This file can then be viewed and analyzed accordingly.

In the present study, we have used frequency statistics from this program to reexamine Novick and Sherman's (2004) data in a regression that includes all relevant bigram statistics, calculated in both type and token forms, as independent variables and solution time as a dependent variable.We have also compared the type and token measures as predictors of lexical decision time, using data from Balota, Cortese, Hutchison, Loftis, Neely, Nelson, and Treiman (2002). This follows the suggestion of Hofmann et al. (2007), who argued that a useful contribution to the type/token controversy "would be to conduct a regression 
analysis with type and token measures as predictors, to find out which measure is most predictive" (Hofmann et al., 2007, p. 623). Lastly, we have examined evidence for the bigram trough hypothesis as an explanation of word identification times.

\section{Results}

Reanalysis of Novick and Sherman (2004) Novick and Sherman (2004) provided solution time and accuracy of solution scores for 108 five-letter anagrams, which they generously made available to us. Since the correlation between the dependent measures is extremely high, $r(106)=.97, p<.0005$, only the reaction time measure will be reported in detail.

The correlations between reaction time to solve the anagram (maximum time allowed of $30 \mathrm{~s}$ ) and all of the bigram frequency measures are shown in Table 1.

It is apparent that neither the token nor the type SBF is significantly correlated with reaction time; nor are they significantly different from each other, $t(105)=0.92$, $p>.05$. Although type top rank is significantly correlated with reaction time, it does not have a significantly higher correlation with reaction time, $t(105)=1.11, p>.05$, than does token top rank. In fact, there are no significant differences between any of the correlations between the various matched token and type measures and reaction time. Furthermore, there were also no significant differences between the matched token and type measures of SBF, $t(105)=.013, p>.05$, and top rank, $t(105)=1.19 p>.05$, with reaction time, when these are calculated using the Novick and Sherman (2004) frequency count and the Mayzner and Tresselt (1965) count.

We conducted a stepwise regression analysis for reaction time with both the top rank variable and BR calculated from the type and token counts and GTZero as independent variables. We used the top rank variable rather than LR, which has a higher correlation with the dependent measure, because it was found to be an important predictorin Novick and Sherman's (2004) earlier analysis of the data. In addition, the type and token versions of the top rank measure are more distinct than are those versions of the LR measure, $r(106)=0.35$ versus $r(106)=.48$, respectively. The most important predictive variable was GTZero, $R=.53, F(1,106)=42.31, p<.001$, with a $\beta=.53$, $t(106)=6.51, p<.001$. The second and only other variable to be entered was BR calculated from the token norms, $R=.58, F(2,105)=26.33$, with a $\beta=.24, t(105)=2.77$, $p<.01$. GTZero has a significantly higher correlation with reaction time than does type top rank, $t(105)=3.15 p<.01$. Thus, it is clear that GTZero is a better predictor of anagram solution times than is type top rank.

Novick and Sherman (2004) pointed out that the Kučera and Francis (1967) word frequencies were based on a subset of English words and would, therefore, be less inclusive than their frequencies based on dictionary definitions. In particular, they argued that a number of ordinary words were omitted that would contribute to people's knowledge of bigram frequencies. We therefore conducted a second stepwise regression in which we included Novick and Sherman's (2004) estimates of top rank, but this did not change the regression equation. Furthermore, GTZero calculated from Solso and Juel (1980) has a significantly higher correlation with anagram solution reaction time than does Novick and Sherman's (2004) top rank measure, $t(105)=2.53, p<.01$, as does GTZero calculated from Mayzner and Tresselt (1965), $t(105)=2.29, p<.05$.

Results also showed that the matched type and token measures were highly correlated when taken from the same corpus of words. In particular, BR, which is one of the more

Table 1 Intercorrelation between Solso and Juel (1980) bigram measures and anagram solution time for Novick and Sherman (2004)

\begin{tabular}{|c|c|c|c|c|c|c|c|c|c|c|}
\hline Variable Words $(n=108)$ & 1 & 2 & 3 & 4 & 5 & 6 & 7 & 8 & 9 & 10 \\
\hline 1 GTZero & - & .13 & .19 & $.43 * *$ & $.42 * *$ & $.28 *$ & $.24 *$ & $.25^{*}$ & $-.39 * *$ & $.53 * *$ \\
\hline 2 Token SBF & & - & $.41 * *$ & $-.60 * *$ & $-.43 * *$ & $-.49 * *$ & $-.28 * *$ & $.46^{* *}$ & $.35 * *$ & -.06 \\
\hline 3 Type SBF & & & - & $-.36^{* *}$ & $-.52 * *$ & $-.35 * *$ & $-.60 * *$ & .22 & $.28 *$ & .04 \\
\hline 4 Token bigramrank & & & & - & $.80 * *$ & $.74 * *$ & $.61^{* *}$ & $-.52 * *$ & $-.47 * *$ & $.43^{* *}$ \\
\hline 5 Typebigram rank & & & & & - & $.52 * *$ & $.85 * *$ & $-.43 * *$ & $-.58 * *$ & $.36 * *$ \\
\hline 6 Token likelihood rank & & & & & & - & $.48^{* *}$ & $-.40 * *$ & $-.32 * *$ & $.26^{*}$ \\
\hline 7 Type likelihood rank & & & & & & & - & $-.24 *$ & $-.40 * *$ & $.26^{*}$ \\
\hline 8 Token top rank & & & & & & & & - & $.35 * *$ & -.13 \\
\hline 9 Type top rank & & & & & & & & & - & $.25^{*}$ \\
\hline 10 Reaction time & & & & & & & & & & - \\
\hline
\end{tabular}

GTZero greater than zero, $S B F$ summed bigram frequency

${ }^{*} p<.01,{ }^{* *} p<.001$ 
important variables for anagram prediction, was correlated at $r(106)=.80, p<.001$, between the token and type measures of Solso and Juel (1980). BR was also highly correlated across corpora. For example, Novick and Sherman's (2004) BR was correlated with Solso and Juel's type BR at $r(106)=.77, p<.001$, and with their token BR at $r(106)=$ $.78, p<.001$. Furthermore, there were no significant differences between any of the matched type and token correlations with solution time when they were taken from the Solso and Juel corpus.

It is interesting to note that the GTZero measure based on frequencies from Mayzner and Tresselt (1965) had a similar correlation, $r(106)=0.52, p<.001$, with solution time as the GTZero based on Solso and Juel (1980). The Mayzner and Tresselt (1965) GTZero was significantly lower $(M=42.06, S D=7.60)$ than the Solso and Juel GTZero $(M=60.08, S D=6.89), t(107)=44.10, p<.0005$; $r(106)=.83, p<.001$, but the correlation with solution time was similar.The larger corpus of words does not seem to improve the quality of prediction of GTZero in this case. It is argued that GTZero is important, since it provides a measure of which bigram combinations and positions can be "ruled out" as possible solutions. Hence, the higher the GTZero, the harder the anagram is to solve. It is worth noting here, however, that the smaller corpus may reflect a lay person's knowledge of words adequately, since a larger corpus may include more unfamiliar words that will suggest that some unlikely bigram positions are permissible.

Our reanalysis of Novick and Sherman (2004) suggested that the distinction between type and token frequencies is of little importance in predicting time for anagram solution. Generalizing the results of language experiments is particularly problematic, however, since a significant result tells us only that the result is likely to generalize to a new set of participants, and not necessarily to a new set of stimuli (Clark, 1973; Coleman, 1964). It was important to demonstrate that these results apply to other anagrams and other indices of anagram difficulty, as well as other participants

Reanalysis of other anagram studies In order to investigate whether similar results would be obtained with different anagrams and different participants, we performed a reanalysis of the results from Gilhooly and Johnson's (1978) study that looked at ease of solution of 80 fiveletter anagrams. This study used the number of participants successfully solving the anagram as the dependent measure. In their original analysis, Gilhooly and Johnson found that starting letter, anagram solution similarity to the target word, pronounce ability of the anagram, and two token bigram frequency measures were most important in determining anagram difficulty.The more important of these two bigram measures was GTZero, which was calculated by hand from a bigram frequency matrix from Mayzner and Tresselt (1965).
Our reanalysis using all relevant measures derived from Solso and Juel (1980) showed that on this occasion, GTZero had the highest correlation with anagram solution score, $r(78)=0.46, p<.005$. Again there were no significant differences between the correlation of any of the matched type and token measures from the same corpus and solution score.

The importance of GTZero and relative unimportance of the type-token distinction can also be seen in other anagram studies. For example, in the Mayzner and Tresselt (1966) study, which looked at solution times for 42 anagrams in six conditions, the correlation between the average time taken to solve an anagram across conditions and GTZero calculated from our program is $r(40)=.35$, $p<.05$. Similarly for Ronning's (1965) study, GTZero has a correlation of $r(18)=.61, p<.02$, with solution time. In every case, the correlation of GTZero and the anagram difficulty measure was higher than the top rank measure, whether calculated from Novick and Sherman (2004) or from Solso and Juel (1980). Furthermore, both the token and typeBR measures were also highly correlated with solution score, and there was never a significant difference between the sizes of the correlation with the dependent measure. In fact,there were no significant differences between any of the matched type and token measures and the dependent variable.

GTZero,derived either from Mayzner and Tresselt (1965) or from our program,was a good predictor of anagram solution, and there were no significant differences between them. Furthermore, the GTZero measure calculated from Novick and Sherman's (2004) frequencies is an equally good predictor. There is, therefore, clear evidence that the distinction between type and token counts has little importance for anagram solution. It is important to note, however, that anagram problems may have limited relevance to word-processing skills. In particular, it is unlikely that GTZero will be a useful variable in predicting performance on other psycholinguistic tasks, since it seems particularly suited to the demands of anagram solution. The next section explores this issue by looking at the relationship of GTZero with a lexical decision task.

Bigram measures and lexical decision making We investigated the relationship of GTZero with reaction time in a lexical decision task for the words employed by Novick and Sherman (2004) and Gilhooly and Johnson (1978). The English Lexicon Project (Balota et al., 2002) provided lexical decision reaction times for 173 words used in the studies above after we removed any duplicates and words for which times were not available. In their lexical decision task (Balota et al., 2002), participants were presented with a string of letters (either a word or a nonword) and were asked to press one button if the string was a word and another button if it was a nonword. As was expected,we 
found no relationship between GTZero and mean lexical decision reaction time, $r(171)=.03, p=.72$.

Given the nature of the lexical decision task, one might expect the LR measure to be a reasonable predictor of lexical decision reaction time. LR indicates the relative frequency of the combination of bigrams in a word, as compared with other combinations. Frequent combinations should, therefore, be most like English words and, therefore, quicker to identify. In this case, the correlation between token LR and reaction time, $r(171)=.29, p<.0005$, was not significantly higher than the type LR correlation with reaction time, $r(171)=.17, p<.05 ; t(170)=1.75$, $p<.10$. Surprisingly the token BR measure has the highest correlation with lexical decision reaction time, $r(171)=.31$, $p<.0005$, which is significantly higher than the correlation between the type BR measure and reaction time, $r(171)=.17$, $p<.05 ; t(170)=3.14, p<.01$. It is also worth noting that this pattern of results would be the same if Solso and Juel's (1980) token measure was compared with a type measure derived from Novick and Sherman (2004).

Overall the correlations between the token measures and lexical decision reaction time were never significantly lower than those of type measures, and sometimes they were significantly higher.

It should also be noted that the correlation between bigram frequencies taken from token and type counts will be fairly high for five-letter words, which makes it unlikely that there would be significant differences between their correlation with other variables. The correlation between token and type measures from Solso and Juel (1980), regardless of word length, is $r(4614)=.41, p<.0001$. The correlation between total token and total type bigram frequency for five-letter words is $r(575)=.75, p<.001$, and it was significantly higher for six-letter words, $r(575)=.95$; $z=13.74, p<.001$.Smaller words, of course, have smaller correlations, since there are bigger discrepancies in their frequencies. For example, the correlation was only $r(577)=.59, p<.0001$, for four-letter words and was significantly lower for three-letter words, $r(577)=.17$, $p<.001 ; z=8.57, p<.001$. Therefore, the distinction between type and token counts might well be more important for words of fewer than five letters.

The bigram trough hypothesis The lexical decision data and our program can also be used to examine the bigram trough hypothesis, which argues that syllable effects are caused by differences in bigram frequency. The number of syllables in a word has been shown to be positively correlated with reaction time in lexical decision tasks, even after important covariates, such as word length, have been controlled (Yap \& Balota, 2009). There was a significant correlation between number of syllables and reaction time, $r(171)=.18, p=.02$, in the present data. If Seidenberg (1987) is correct, the syllable effect should be caused by the presence of bigram troughs in multisyllabic words. It is surprising, however, that there is quite a large number of one-syllable words $(57 / 114)$ that have a bigram trough as defined by Rapp (1992). For example, in the word blush, there is a trough between the first and third bigrams; the first bigram $b l$ has a frequency of 687 , the second bigram $l u$ only has a frequency of 205 , and the third bigram ushas a frequency of 919 . There are also quite a few multisyllablic words that do not have a bigram trough (17/59). For example, in the word basic; the first bigram $b a$ has a frequency of 812 , the second bigram as has a frequency of 939, the third bigram si has a frequency of 714 , and the fourth bigram ic has a frequency of 595. It is true that multisyllabic words are more likely to have a trough, $\mathrm{X}^{2}(1$, $N=173)=7.02, p=.01$, but the large number of onesyllable words with a trough makes it unlikely that it can be an explanation of the syllable number effect.

So far, research examining the bigram trough hypothesis as a cause of the various syllable effects in reading has focused on multisyllabic stimuli (Conrad, Carreiras, Tamm, \& Jacobs, 2009; Rapp, 1992). It is clear, however, that if such effects are caused by relative bigram frequencies, they should also occur in one-syllable words with troughs.In this case, there was no significant difference in reaction times between one-syllable words with and without troughs, $t(112)=1.12, p=.26$. There was also no significant difference in reaction times between two-syllable words with and without troughs, $t(57)=0.60$, $p=.55$. The latter result offers some support to the recent finding by Conrad et al. (2009) that syllable frequency effects in bisyllabic Spanish words were unaffected by the presence or absence of a bigram trough.

\section{Discussion}

We have presented a program that will calculate GTZero, BR, LR, and SBF from both type- and token-based systems simultaneously, using the Solso and Juel (1980) frequencies. This program will perform these calculations on any word or letter string from two to nine letters long. This program extends previous research that has provided a program for the calculation of some of these bigram statistics from a type-based system for five-letter words (Novick \& Sherman, 2004).

Our reanalysis of previous research using this program has shown that GTZero and BR calculated from either a type or a token count are the most important bigram frequency variables in determining the difficulty of anagrams. We have also shown that there is no evidence that type-based systems will be superior in predicting anagram difficulty.

We have demonstrated that this program will be useful not just in anagram research, but also in areas of visual word recognition. In particular, we have shown that the 
distinction between type and token bigram frequencies has little importance when word identification reaction times are predicted. In fact, in our analysis, which uses all of the matched token and type frequencies, the token measure of BR was the best predictor of word identification reaction times and was significantly better than the type measure of BR derived either from Novick and Sherman (2004) or from Solso and Juel (1980). This is particularly important because although anagram solution may be regarded as an unusual task, lexical decision has been described as a "defacto gold standard in visual word recognition research" (Yap \& Balota, 2009, p. 502).

Lastly, we used the program to investigate the bigram trough explanation of the syllable number effect. Previous research in English on the bigram trough hypothesis has used frequencies derived by hand from the Solso and Juel (1980) tables, which, as we noted, is a time-consuming process. In this research, only multisyllabic words have been investigated, even though monosyllabic words also have troughs, as we have shown. It was, in fact, surprising that so many monosyllabic words in our sample had a bigram trough, and this makes it unlikely that syllable effects are caused by troughs. Clearly, if it is the presence or absence of a bigram trough that causes syllable effects, onesyllable words with troughs should have longer lexical decision reaction times than should those without troughs. We found, however, that there were no significant differences in lexical decision time between words with and without troughs for either mono- or polysyllabic words. It would be important to demonstrate this again with other variables considered, such as consonant-vowel structure. Our program makes it easy to include variables such as a bigram trough and its relative position in any future studies of syllabic or other effects in word processing. We believe that our program should be useful for all research that looks at the impact of sublexical features on visual word recognition and reading processes (Aichert \& Ziegler, 2005).

Author note We thank Prof. Laura Novick of Vanderbilt University and Prof. Jim Sherman of Indiana University for generously allowing us to reanalyze their data.

\section{References}

Aichert, I., \& Ziegler, W. (2005). Is there a need to control for sublexical frequencies? Brain and Language, 95, 170-171.

Balota, D. A., Cortese, M.J., Hutchison, K.A., Loftis, B., Neely, J.H., Nelson, D., . . Treiman, R. (2002). The English Lexicon Project: A Web-based repository of descriptive and behavioral measures for 40,481 English Words and Nonwords, St. Louis: Washington University.Retrieved from http://elexicon.wustl.edu/
Clark, H. H. (1973). The language as fixed-effect fallacy: A critique of language statistics in psychological research. Journal of Verbal Learning and Verbal Behaviour, 12, 335-339.

Coleman, E. B. (1964). Generalizing to a language population. Psychological Reports, 14, 219-226.

Conrad, M., Carreiras, M., \& Jacobs, A. M. (2008). Contrasting effects of token and type syllable frequency in lexical decision. Language and Cognitive Processes, 23, 296-326.

Conrad, M., Carreiras, M., Tamm, S., \& Jacobs, A. M. (2009). Syllables and bigrams: Orthographic redundancy and syllabic units affect visual word recognition at different processing levels. Journal of Experimental Psychology: Human Perception and Performance, 35, 461-479.

Gilhooly, K., \& Johnson, C. E. (1978). Effects of solution word attributes on anagram difficulty: A regression analysis. The Quarterly Journal of Experimental Psychology, 30, 57-70.

Hofmann, M. J., Stenneken, P., Conrad, M., \& Jacobs, A. M. (2007). Sublexical frequency measures of orthographic and phonological units in German. Behavior Research Methods, Instruments, \& Computers, 36, 397-401.

Kučera, H., \& Francis, W. (1967). Computational analysis of present-day American English. Providence: Brown University Press.

Mayzner, M. S., \& Tresselt, M. E. (1959). Anagram solution times: A function of transition probabilities. The Journal of Psychology, 47, 117-125.

Mayzner, M. S., \& Tresselt, M. E. (1965). Tables of single-letter and bigram frequency counts for various word length and letter position combinations. Psychonomic Monograph Supplement, 1, 13-31.

Mayzner, M. S., \& Tresselt, M. E. (1966). Anagram solution times: A function of multiple- solution anagrams. Journal of Experimental Psychology, 71, 66-73.

Mendelsohn, G. A. (1976). An hypothesis approach to the solution of anagrams. Memory \& Cognition, 4, 637-642.

Mendelsohn, G. A., \& O’Brien, A. T. (1974). The solution of anagrams: A reexamination of the effects of letter transition probabilities, letter moves, and word frequency on anagram difficulty. Memory \&Cognition, 3, 566-574.

Novick, L. R., \& Sherman, S. J. (2004). Type-based bigram frequencies for five-letter words. Behavior Research Methods, Instruments, \&Computers, 36, 397-401.

Novick, L. R., \& Sherman, S. J. (2008). The effects of superficial and structural information on on-line problem solving for good versus poor anagram solvers. The Quarterly Journal of Experimental Psychology, 61, 1098-1120.

Rapp, B. (1992). The nature of sub-lexical orthographic organization: The bigram trough hypothesis examined. Journal of Memory and Language, 25, 461-475.

Ronning, R. R. (1965). Anagram solution times: A function of the "ruleout" factor. Journal of Experimental Psychology, 69, 35-39.

Seidenberg, M. S. (198). Reading complex words. In G. Carlson \& M. Tannenhaus (Eds.), Linguistic structure in language processing (pp. 53-105). Dordrecht: Kluver Academic.

Seidenberg, M. S. (1987). Sublexical structures in visual word recognition: Access units or orthographic redundancy? In $\mathrm{M}$. Coltheart (Ed.), Attention and performance XII: The psychology of reading (pp. 245-263). Hillsdale: Erlbaum.

Solso, R. L., \& Juel, C. L. (1980). Positional frequency and versatility of bigrams for two- through nine-letter English words. Behavior Research Methods \& Instrumentation, 12, 297-343.

Solso, R. L., Topper, G. E., \& Macey, W. H. (1973). Anagram solution as a function of bigram versatility. Journal of Experimental Psychology, 100, 259-262.

Yap, M. J., \& Balota, D. A. (2009). Visual word recognition of multisyllabic words. Journal of Memory and Language, 60, 502-509. 